\title{
The Apoptotic Effect of the Methanol Extract of Polygonum cuspidatum through Up-Regulation Death Receptor 5 and CHOP in HSC-2 Human Oral Cancer Cells
}

\author{
Hyun-Ju Yu ${ }^{1}$, Ji-Ae Shin ${ }^{1}$, Eun-Sun Choi ${ }^{1}$, Jae-Gyu Jeon ${ }^{2}$, Nam-Pyo Cho ${ }^{1}$, Sung-Dae Cho ${ }^{1 *}$ \\ ${ }^{1}$ Department of Oral Pathology, School of Dentistry, Institute of Oral Bioscience, Chonbuk National University, Jeonju, Korea; \\ ${ }^{2}$ Department of Preventive Dentistry, School of Dentistry, Institute of Oral Bioscience, Chonbuk National University, Jeonju, Korea. \\ Email: ${ }^{*}$ efiwdsc@chonbuk.ac.kr
}

Received January $1^{\text {st }}, 2012$; revised February $2^{\text {nd }}, 2012$; accepted February $12^{\text {th }}, 2012$

\begin{abstract}
Polygonum cuspidatum is used as a traditional medicinal herb for the therapy of various diseases including several types of cancers. In the present study, we focused on addressing the anti-cancer activity and molecular mechanism of methanol extract of Polygonum cuspidatum (MEPC) in HSC-2 human oral cancer cells. The effect of MEPC on oral cancer cells was estimated by 3-(4,5-dimethylthiazol-20yl)-(3-carboxymethoxyphenyl)-2-(4-sulphophenyl)-2H-tetrazolium (MTS) assay, 4'-6-diamidino-2-phenylindole (DAPI) staining and Western blot analysis. MEPC inhibited the cell viability and induced apoptosis through the induction of death receptor 5 (DR5). MEPC also increased the expression of C/EBP homologous protein/growth arrest and the DNA damage-inducible gene 153 (CHOP), a transcription factor induced by ER stress. Thus, we concluded that the induction of CHOP leading to DR5 up-regulation is required for the anti-cancer activity of MEPC in HSC-2 cells and MEPC may be a promising drug candidate for oral cancer.
\end{abstract}

Keywords: Polygonum cuspidatum; Endoplasmic Reticulum Stress; C/EBP Homologous Protein/Growth Arrest and the DNA Damage-Inducible Gene 153 (CHOP); Death Receptor 5 (DR5); Apoptosis; Human Oral Cancer Cell

\section{Introduction}

Oral cancer is one of the leading causes of cancer-related deaths in developed countries [1], and it is estimated that 36,540 new cases diagnosed in the United States in last year (2010) [2]. Several factors are associated with increased risk of oral cancer, and these include smoking and alcohol usage [3-5]. Because oral cancer is frequently not detected in early stages, oral cancer patients have a relatively low five-year survival rate [2]. Therefore, the clinical trials such as early detection and therapeutic strategies for oral cancer are still required.

The function of the endoplasmic reticulum (ER) is essential for cellular activities and survival in eukaryotic cells. The ER is the primary organelle for protein synthesis, initial posttranslational modification, proper protein folding and assembling of secreted, membrane-bound and some organelle-targeted proteins [6,7]. The accumulation of misfolded and unfolded protein in ER lumen results in the condition referred to as ER stress, and then leads to ER stress response known as unfolded protein response (UPR). To resolve this problem, cells evolved various

\footnotetext{
${ }^{*}$ Corresponding author.
}

protective strategies such as transcriptional induction of chaperone genes for protein folding, translational attenuation of protein synthesis and protein degradation by proteasome [8,9]. However, if misfolded and unfolded protein aggregation is persistent and cannot be resolved, this response also results in apoptosis. Earlier studies showed that ER stress-induced apoptosis up-regulates the transcription factor C/EBP homologous protein/growth arrest and the DNA damage-inducible gene 153 (CHOP/ GADD153) [10,11]. In addition, induction of DR5 through activation of CHOP plays an important role in ER-stress induced apoptosis [12-18].

The naturally occurring products derived from plant sources are used as traditional medicines due to their potential chemotherapeutic activity. Polygonum cuspidatum, a traditional medicinal herb, has been used for the therapy of various diseases including inflammation, hepatitis and diarrhea [19-23]. In many previous studies, Polygonum cuspidatum was found that it contains several constituents such as resveratrol, emodin, polydatin, physicon and rhein [24-26]. The extract of Polygonum cuspidatum including these constituents have been reported to possess an important role for anti-inflammatory 
and anti-cancer activities [21,27-30]. In particular, our group reported that emodin in Polygonum cuspidatum has anti-proliferative and pro-apoptotic effects in several oral cancer cells and $\mathrm{Sp} 1$ and survivin played important role for P. cuspidatum-induced apoptosis [31]. However, the apoptotic effect of Polygonum cuspidatum in oral cancer cells related to ER stress is not well studied yet.

In the present study, we investigated the growth-inhibitory effect of MEPC in oral cancer and the molecular target for MEPC-induced apoptosis. The results showed that MEPC induced apoptosis and it modulated the expressions of DR5 and CHOP in HSC-2 human oral cancer cells.

\section{Materials and Methods}

\subsection{Reagents and Antibodies}

The methanol extracts of Polygonum cuspidatum (MEPC) was kindly given by Dr. Jeon in Chonbuk National University (Jeonju, Korea). Dulbecco's modified essential medium (DMEM), fetal bovine serum (FBS), 100× antibiotic solution, Trypsin, and D-PBS was obtained from WelGENE Inc. (Dae-gu, Korea). The Poly (ADP-ribose) polymerase (PARP) antibody was purchased from BD Pharmingen $^{\text {TM }}$ (San Jose, CA, USA). Antibodies recognizing cleaved caspase-3 (Asp175) and DR5 were purchased from Cell Signaling Technology (Denver, MA, USA). The antibodies for CHOP (Cat No. sc-793; R-20) and actin (Cat No. sc-1615; C-11) were purchased from Santa Cruz Biotechnology (Santa Cruz, CA, USA). DR4 antibody was obtained from R\&D Systems Inc. (Minneapolis, MN, USA). Cell Titer $96^{\circledR}$ Aqueous One Solution Cell Proliferation assay kit for 3-(4,5-dimethylthiazol-20yl)-(3-carboxymethoxyphenyl)-2-(4-sulphophenyl)$2 \mathrm{H}$-tetrazolium) (MTS) assay was purchased from Promega (Madison, WI, USA). DAPI (4'-6-Diamidino-2phenylindole) was obtained from Sigma-Aldrich Inc. (St. Louis, MO, USA).

\subsection{Cell Culture and Chemical Treatment}

HSC-2 cells were obtained from Prof. Shindo in Hokkaido University (Hokkaido, Japan). HSC-2 cells were cultured in DMEM supplemented with $10 \%$ FBS and $100 \mathrm{U} / \mathrm{ml}$ each of penicillin and streptomycin in a humidified atmosphere containing $5 \% \mathrm{CO}_{2}$ at $37^{\circ} \mathrm{C}$. An equal number of cells were seeded and allowed to attach. When the cells reached $50 \%$ confluence, the cells were treated with DMSO or MEPC $(25,50$ and $100 \mathrm{~g} / \mathrm{ml})$. MEPC was dissolved in $0.1 \%$ DMSO (vehicle control)

\subsection{MTS Assay}

The effects of MEPC on cell viability were estimated using the Cell Titer 96 Aqueous One Solution Cell Proliferation Assay kit. The cells were seeded in a 96-well plate and then incubated for $24 \mathrm{hr}$. After MEPC treatment for 24 and $48 \mathrm{hr}$, a MTS solution was added to each well and the plates were incubated at $37^{\circ} \mathrm{C}$ in humidified $5 \% \mathrm{CO}_{2}$ atmosphere for $2 \mathrm{hr}$. The absorbance at $490 \mathrm{~nm}$ was recorded using ELISA plate reader (BIO-TEK Instruments, Inc., Madison, WI, USA). The data were expressed as the percentage of cell viability compared to the vehicle control.

\subsection{DAPI Staining}

Apoptotic cell death was determined morphologically using a fluorescent nuclear dye, DAPI staining. HSC-2 cells were incubated with DMSO or MEPC $(25,50$ and $100 \mathrm{~g} / \mathrm{ml}$ ) for $48 \mathrm{hr}$, HSC-2 cells were harvested by the trypsinization and fixed in $70 \%$ ethanol overnight at $4 \mathrm{C}$. The cells were re-suspended in PBS, deposited on polyL-lysin coated slides, stained with a DAPI solution, deposited onto the slides and finally viewed to detect apoptotic characteristics with a fluorescent microscope.

\subsection{Western Blot Analysis}

Whole-cell lysates were extracted and quantified with DC Protein Assay kit (BIO-RAD, Hercules. CA, USA). Equal amount of protein from each sample was mixed with $5 \mathrm{X}$ loading buffer and heated at $95^{\circ} \mathrm{C}$ for $5 \mathrm{~min}$. Equal amount of protein was separated by SDS-polyacrylamide gel electrophoresis (PAGE) and transferred onto polyvinylidene fluoride (PVDF) membrane (BIORAD, Hercules. CA, USA). The membranes was blocked with 5\% skim milk in TBST buffer at RT for $2 \mathrm{hr}$, washed with TBST and maintained overnight at $4^{\circ} \mathrm{C}$ with primary antibody. Washing was done with TBST and incubation with horseradish peroxidase (HRP)-conjugated secondary antibody was done at RT for $2 \mathrm{hr}$. After washing with TBST, the antibody-bound proteins were detected using an ECL Western Blotting Luminol Reagent (Santa Cruz, CA, USA).

\subsection{Statistical Analysis}

The data were assessed for statistical significance using a Student's t-test. A value of $p<0.05$ compared with the solvent control was considered statistically significant.

\section{Results}

\subsection{MEPC Inhibits the Cell Viability and Induces Apoptosis in HSC-2 Human Oral Cancer Cells}

The effect of MEPC on the viability of HSC-2 cells was investigated using the MTS assay. The HSC-2 cells were 
treated with various doses of $\operatorname{MEPC}(25,50$ and 100 $\mu \mathrm{g} / \mathrm{ml}$ ) for 24 and $48 \mathrm{hr}$. The result shown in Figure 1(a) indicated that the viability of HSC-2 cells significantly inhibited upon treatment with MEPC in dose-dependent manner. To evaluate why MEPC inhibits the cell viability, we next investigated the apoptotic activity of MEPC by Western blot analysis. As shown in Figure 1(b), MEPC-treated cells showed decreased total PARP and activated caspase- 3 than control cells. We also examined the apoptotic activity of MEPC by DAPI staining. As shown in Figure 2, nuclear staining with DAPI revealed that nuclear condensation and fragmentation exhibited in the cells treated with higher doses of MEPC for $48 \mathrm{hr}$. These results show that MEPC has anti-proliferative and apoptotic effect in HSC-2 cells.

\subsection{MEPC Increases DR5 Expression, but Not DR4}

To further investigate that whether MEPC-induced apoptosis is involved in extrinsic pathway by death receptors, the protein levels of death receptor 4 and 5 were detected. As shown in Figure 3(a), MEPC increased the expression of DR5 in dose-dependent manner compared to vehicle control, but did not affect DR4 expression. Based on these results, we performed experiments in triplicate. As

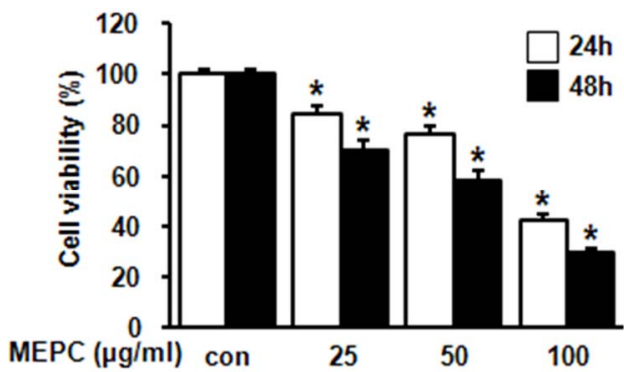

(a)

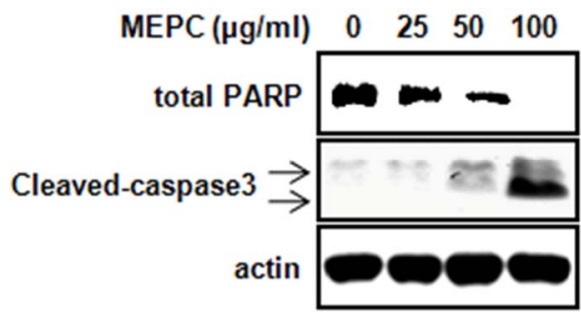

(b)

Figure 1. HSC-2 cell were treated with DMSO or various doses of methanol extract of Polygonum cuspidatum (MEPC). (a) Cell viability was determined using the MTS assay in HSC-2 cells for 24 and $48 \mathrm{hr}$. The bars are the means \pm SD of triplicate experiments. ${ }^{*}, p<0.05$ compared to the vehicle control group; (b) The effect of MEPC on the total PARP and cleaved caspase- 3 in HSC-2 cells was determined by Western blot analysis. Actin was used to normalize the protein loading from each treatment.

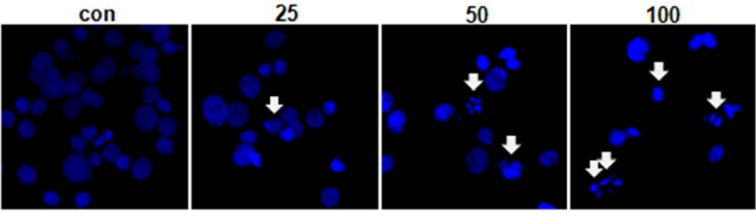

(a)

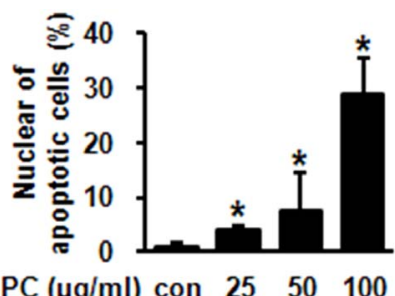

(b)

Figure 2. (a) HSC-2 cells were treated with DMSO or various doses of MEPC for $48 \mathrm{hr}$. Cells were harvested and the apoptotic effect of MEPC in HSC-2 cells was determined by DAPI staining. DNA fragmentation and nuclear condensation were detected by fluorescence microscopy (Magnification, $\times 400)$; (b) The apoptotic cells were counted and the data shown in the graphs expressed the means \pm SD of triplicate experiments. $*, p<0.05$ compared to the vehicle control group.

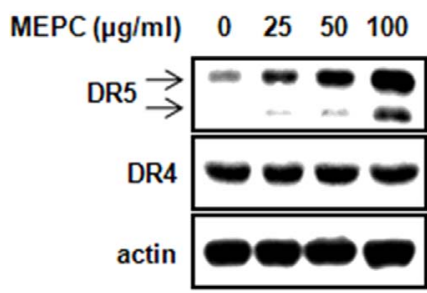

(a)

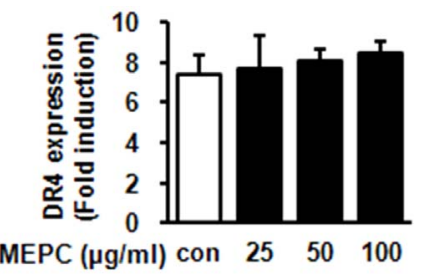

(b)

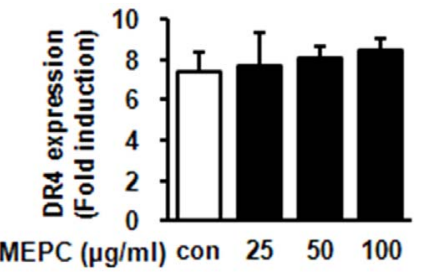

(c)

Figure 3. HSC-2 cells were treated with DMSO or 25, 50 and $100 \mu \mathrm{g} / \mathrm{ml}$ of MEPC for $48 \mathrm{hr}$. (a) The protein levels of DR5 and DR4 were analyzed by Western blot analysis; (b) and (c) The protein levels of DR5 and DR4 were confirmed in triplicate experiments, and then the graphs expressed the means $\pm \mathrm{SD}, *, p<0.05$ compared to the vehicle control group. 
shown in Figures 3(b) and (c), HSC-2 cells treated with $100 \mu \mathrm{g} / \mathrm{ml}$ of MEPC increased DR5 expression up to 12 folds compared to vehicle control whereas MEPC did not cause significant change in DR4 expression. These results showed that the extrinsic pathway by DR5 contributed to MEPC-induced apoptosis in HSC-2 cells.

\subsection{MEPC Up-Regulates CHOP Expression through Endoplasmic Reticulum Stress}

In previous studies, it was reported that the up-regulation of CHOP by ER stress was required for DR5 expression $[12,13]$. Thus, we examined whether MEPC affected the expression of CHOP, transcription factor related to ER stress. The result shown in Figure 4(a) indicated that MEPC-treated cells increased CHOP expression compared to vehicle control. Based on these results, we performed experiments in triplicate. As shown in Figure 4(b), cells treated with $100 \mu \mathrm{g} / \mathrm{ml}$ of MEPC increased CHOP expression up to 6 folds. Therefore, these results suggest that the up-regulation of CHOP was involved in MEPC-induced apoptosis.

\section{Discussion}

The naturally occurring products derived from plant are abundant source of bioactive constituents that have played an important role in pharmacology. Based on traditional therapeutic applications, several natural products have been demonstrated their beneficial effects in various cancer cells. Selaginella tamariscina, an oriental medicinal plant, induced p53 expression and G1 arrest, and suggests that this might contribute to cytotoxic effects by causing apoptotic DNA fragmentation in human leukemia

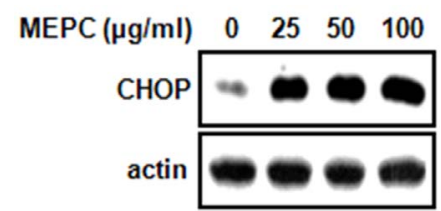

(a)

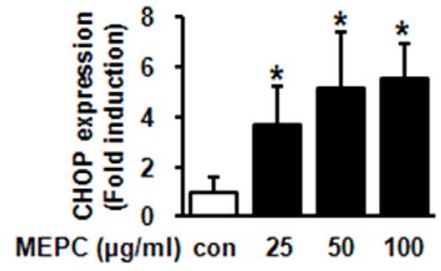

(b)

Figure 4. (a) The protein level of CHOP was analyzed by Western blot analysis in HSC-2 cells treated with various doses of MEPC for $48 \mathrm{hr}$; (b) The protein level of CHOP was confirmed in triplicate experiments, and then the graphs expressed the means \pm SD. ${ }^{*}, p<0.05$ compared to the vehicle control group. cells and human ovarian cancer cells [32]. Other study also reported that the extract of Alpinia oxyphylla inhibited the cell proliferation of skin cancer cells by inducting apoptosis [33]. In addition, the extract of Solanum muricatum suppressed tumor growth both in vivo and in vitro by inducing apoptosis [34]. These studies showed that the products derived from naturally occurring plants are useful for the prevention of cancer. In particular, chemopreventive agents such as naturally occurring product consist of a diverse group of compounds with different mechanisms of action, but, their ability to induce apoptosis may represent a unifying concept for the mechanism of chemoprevention [35]. Therefore, these studies that explain their ability to induce apoptosis and mechanism of action are valuable for their clinical application in prevention and therapy of cancer.

In the present study, we examined the effect of MEPC on the growth inhibition of HSC-2 human oral cancer cells and what kind of key molecular target is involved in MEPC-induced apoptosis. We found that MEPC clearly decreased the number of viable cells in a dose-dependent manner and induced apoptosis as evidenced by total PARP decrease, and activation of caspase- 3 in HSC-2 oral cancer cells (Figure 1). We also found that MEPC increases the nuclear condensation and fragmentation (Figure 2). These results suggest that MEPC induces apoptosis to inhibit the growth of oral cancer cells. Because death receptors (DR4 and DR5) are essential for extrinsic pathway of apoptosis, targeting death receptor-mediated apoptosis is effective strategy for prevention of cancer. Previous studies reported that Honokiol, a natural product purified from magnolia, is effective sensitizer of death receptormediated apoptosis in lung cancer cells [36]. Dihydroflavonol derived from Blumea balsamifera, are also known to induce apoptosis through DR5 up-regulation in leukemia cells [37]. Thus, we investigated whether MEPC can cause the expression of death receptors in oral cancer cells. The data showed that MEPC clearly increased DR5 expression, but did not affect DR4 expression (Figure 3). These results suggest that MEPC stimulates the extrinsic pathway by DR5.

ER stress is an important pathway that responds to ER dysfunction such as accumulation of misfolded and unfolded protein. The expression of genes associated with ER stress, including CHOP, GRP78 and ATF4, is increased by ER stress responses $[6,8,9]$. In particular, CHOP, an important marker for ER stress, is up-regulated in ER stress-induced apoptosis [16,38]. Interestingly, earlier studies suggested that ER-stress induced apoptosis through up-regulation of $\mathrm{CHOP}$ is associated with induction of the DR5 expression $[12,13,16]$. Our recent study in several oral cancer cells also show that C-DIMs activate the ER stress pathway leading to the 
up-regulation of DR5 and cleaved Bid and caspase 8 [39]. In this study, MEPC potently stimulated the expression of CHOP (Figure 4), suggesting that up-regulation of CHOP contributes to MEPC-induced apoptosis.

\section{Conclusion}

In conclusion, this study showed that MEPC decreased cell viability and induced apoptosis through the induction of DR5 in HSC-2 oral cancer cells. Moreover, MEPC induced CHOP expression, a typical marker for ER stress. Taken together, we suggest that MEPC-induced apoptosis through the induction of DR5 might be mediated by ER stress leading to the up-regulation of CHOP in oral cancer cells, and MEPC may have a clinical potential for effective treatment of oral cancer although we need to investigate the anti-cancer efficacy and toxicity of MEPC in the in-vivo animal model.

\section{REFERENCES}

[1] G. J. Macfarlane, P. Boyle, T. V. Evstifeeva, C. Robertson and C. Scully, "Rising Trends of Oral Cancer Mortality among Males Worldwide: The Return of an Old Public Health Problem," Cancer Causes and Control, Vol. 5, No. 3, 1994, pp. 259-265. doi:10.1007/BF01830246

[2] A. Jemal, R. Siegel, J. Xu and E. Ward, "Cancer Statistics, 2010," CA: A Cancer Journal for Clinical, Vol. 60, No. 5, 2010, pp. 277-300. doi:10.3322/caac.20073

[3] A. G. Zygogianni, G. Kyrgias, P. Karakitsos, A. Psyrri, J. Kouvaris, N. Kelekis and V. Kouloulias, "Oral Squamous Cell Cancer: Early Detection and the Role of Alcohol and Smoking," Head \& Neck Oncology, Vol. 3, 2011, p. 2. doi:10.1186/1758-3284-3-2

[4] A. Zygogianni, G. Kyrgias, K. Mystakidou, C. Antypas, J. Kouvaris, C. Papadimitriou, V. Armonis, H. Alkati and V. Kouloulias, "Potential Role of the Alcohol and Smoking in the Squamous Cell Carcinoma of the Head and Neck: Review of the Current Literature and New Perspectives," Asian Pacific Journal of Cancer Prevention, Vol. 12, No. 2, 2011, pp. 339-344.

[5] O. I. Mansour, C. H. Snyderman and F. D'Amico, "Association between Tobacco Use and Metastatic Neck Disease," Laryngoscope, Vol. 113, No. 1, 2003, pp. 161-166. doi:10.1097/00005537-200301000-00030

[6] E. Szegezdi, S. E. Logue, A. M. Gorman and A. Samali, "Mediators of Endoplasmic Reticulum Stress-Induced Apoptosis," EMBO Reports, Vol. 7, No. 9, 2006, pp. 880885. doi:10.1038/sj.embor.7400779

[7] C. Xu, B. Bailly-Maitre and J. C. Reed, "Endoplasmic Reticulum Stress: Cell Life and Death Decisions," The Journal of Clinical Investigation, Vol. 115, No. 10, 2005, pp. 2656-2664. doi:10.1172/JCI26373

[8] E. Araki, S. Oyadomari and M. Mori, "Impact of Endoplasmic Reticulum Stress Pathway on Pancreatic BetaCells and Diabetes Mellitus," Experimental Biology and Medicine, Vol. 228, No. 10, 2003, pp. 1213-1217.
[9] M. J. Gething, "Role and Regulation of the ER Chaperone BiP," Seminars in Cell \& Developmental Biology, Vol. 10, No. 10, 1999, pp. 465-472. doi:10.1006/scdb.1999.0318

[10] R. J. Kaufman, "Stress Signaling from the Lumen of the Endoplasmic Reticulum: Coordination of Gene Transcriptional and Translational Controls," Genes Development, Vol. 13, No. 10, 1999, pp. 1211-1233. doi:10.1101/gad.13.10.1211

[11] S. Moriya, K. Miyazawa, T. Kawaguchi, X. F. Che and A. Tomoda, "Involvement of Endoplasmic Reticulum StressMediated CHOP (GADD153) Induction in the Cytotoxicity of 2-Aminophenoxazine-3-one in Cancer Cells," International Journal of Oncology, Vol. 39, No. 4, 2011, pp. 981-988.

[12] M. Abdelrahim, K. Newman, K. Vanderlaag, I. Samudio and S. Safe, "3,3'-Diindolylmethane (DIM) and Its Derivatives Induce Apoptosis in Pancreatic Cancer Cells through Endoplasmic Reticulum Stress-Dependent Upregulation of DR5," Carcinogenesis, Vol. 27, No. 4, 2006, pp. 717 728. doi:10.1093/carcin/bgi270

[13] W. Zou, P. Yue, F. R. Khuri and S. Y. Sun, "Coupling of Endoplasmic Reticulum Stress to CDDO-Me-Induced UpRegulation of Death Receptor 5 via a CHOP-Dependent Mechanism Involving JNK Activation," Cancer Research, Vol. 68, No. 18, 2008, pp. 7484-7492. doi:10.1158/0008-5472.CAN-08-1318

[14] P. Lei, M. Abdelrahim, S. D. Cho, X. Liu and S. Safe, "Structure-Dependent Activation of Endoplasmic Reticulum Stress-Mediated Apoptosis in Pancreatic Cancer by 1,1-bis(3'-indoly)-1-(p-substituted phenyl) methanes," Molecular Cancer Therapeutics, Vol. 7, No. 10, 2008, pp. 3363-3372. doi:10.1158/1535-7163.MCT-08-0439

[15] S. Chen, X. Liu, P. Yue, A. H. Schonthal, F. R. Khuri and S. Y. Sun, "CCAAT/Enhancer Binding Protein Homologous Protein-Dependent Death Receptor 5 Induction and Ubiquitin/Proteasome-Mediated Cellular FLICE-Inhibitory Protein Down-Regulation Contribute to Enhancement of Tumor Necrosis Factor-Related Apoptosis-Inducing Ligand-Induced Apoptosis by Dimethyl-Celecoxib in $\mathrm{Hu}-$ man Non Small-Cell Lung Cancer Cells," Molecular Pharmacology, Vol. 72, No. 5, 2007, pp. 1269-1279. doi:10.1124/mol.107.037465

[16] H. Yamaguchi and H. G. Wang, "CHOP Is Involved in Endoplasmic Reticulum Stress-Induced Apoptosis by Enhancing DR5 Expression in Human Carcinoma Cells," Journal of Biological Chemistry, Vol. 279, No. 44, 2004, pp. 45495-45502. doi:10.1074/jbc.M406933200

[17] T. Yoshida, T. Shiraishi, S. Nakata, M. Horinaka, M. Wakada, Y. Mizutani, T. Miki and T. Sakai, "Proteasome Inhibitor MG132 Induces Death Receptor 5 through CCAAT/ Enhancer-Binding Protein Homologous Protein," Cancer Research, Vol. 65, No.13, 2005, pp. 5662-5667. doi:10.1158/0008-5472.CAN-05-0693

[18] S. Y. Lee, M. S. Lee, R. P. Cherla and V. L. Tesh, "Shiga Toxin 1 Induces Apoptosis through the Endoplasmic Reticulum Stress Response in Human Monocytic Cells," Cellular Microbiology, Vol. 10, No. 3, 2008, pp. 770-780. doi:10.1111/j.1462-5822.2007.01083.x 
[19] J. S. Chang, H. W. Liu, K. C. Wang, M. C. Chen, L. C. Chiang, Y. C. Hua and C. C. Lin, "Ethanol Extract of Polygonum cuspidatum Inhibits Hepatitis B Virus in a Stable HBV-Producing Cell Line," Antiviral Research, Vol. 66, No. 1, 2005, pp. 29-34. doi:10.1016/j.antiviral.2004.12.006

[20] J. Choi, C. C. Conrad, C. A. Malakowsky, J. M. Talent, C. S. Yuan and R. W. Gracy, "Flavones from Scutellaria baicalensis Georgi Attenuate Apoptosis and Protein Oxidation in Neuronal Cell Lines," Biochimica et Biophysica Acta, Vol. 1571, No. 3, 2002, pp. 201-210. doi:10.1016/S0304-4165(02)00217-9

[21] L. Feng, L. F. Zhang, T. Yan, J. Jin and W. Y. Tao, "Studies on Active Substance of Anticancer Effect in Polygonum cuspidatum," Zhong Yao Cai, Vol. 29, No. 7, 2006, pp. 689-691.

[22] H. Ghanim, C. L. Sia, S. Abuaysheh, K. Korzeniewski, P. Patnaik, A. Marumganti, A. Chaudhuri and P. Dandona, "An Antiinflammatory and Reactive Oxygen Species Suppressive Effects of an Extract of Polygonum cuspidatum Containing Resveratrol," Journal of Clinical Endocrinology \& Metabolism, Vol. 95, No. 9, 2010, pp. E1-E8. doi:10.1210/jc.2010-0482

[23] Y. Kimura and H. Okuda, "Resveratrol Isolated from Polygonum cuspidatum Root Prevents Tumor Growth and Metastasis to Lung and Tumor-Induced Neovascularization in Lewis Lung Carcinoma-Bearing Mice," Journal of Nutrition, Vol. 131, No. 6, 2001, pp. 1844-1849.

[24] J. Dong, H. Wang, L. Wan, Y. Hashi and S. Chen, "Identification and Determination of Major Constituents in Polygonum cuspidatum Sieb. et Zucc. by High Performance Liquid Chromatography/Electrospray Ionization-Ion Trap-Time-of-Flight Mass Spectrometry," Se Pu, Vol. 27, No. 4, 2009, pp. 425-430.

[25] L. Xue, "Progress in the Pharmacological Study of Chinese Herbal Drug: Polygonum cuspidatum," Zhongguo Zhong Yao Za Zhi, Vol. 25, No. 11, 2000, pp. 651-653.

[26] Y. L. Leu, T. L. Hwang, J. W. Hu and J. Y. Fang, “Anthraquinones from Polygonum cuspidatum as Tyrosinase Inhibitors for Dermal Use," Phytotherapy Research, Vol. 22, No. 4, 2008, pp. 552-556. doi:10.1210/jc.2010-0482

[27] S. Banerjee, C. Bueso-Ramos and B. B. Aggarwal, "Suppression of 7,12-Dimethylbenz(a) Anthracene-Induced Mammary Carcinogenesis in Rats by Resveratrol: Role of Nuclear Factor- $\kappa B$, Cyclooxygenase 2 and Matrix Metalloprotease 9," Cancer Research, Vol. 62, No. 17, 2002, pp. 4945-4954.

[28] A. Kumar, S. Dhawan and B. B. Aggarwal, "Emodin (3Methyl-1,6,8-trihydroxyanthraquinone) Inhibits TNF-In-

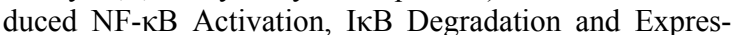
sion of Cell Surface Adhesion Proteins in Human Vascular Endothelial Cells," Oncogene, Vol. 17, No. 7, 1998, pp. 913-918. doi:10.1038/sj.onc. 1201998

[29] S. H. Lee, S. Y. Ryu, H. B. Kim, M. Y. Kim and Y. J. Chun, "Induction of Apoptosis by 3,4'-Dimethoxy-5-hydroxystilbene in Human Promyeloid Leukemic HL-60 Cells," Planta Medicine, Vol. 68, No. 2, 2002, pp. 123127. doi:10.1055/s-2002-20242
[30] Y. W. Lin, F. J. Yang, C. L. Chen, W. T. Lee and R. S. Chen, "Free Radical Scavenging Activity and Antiproliferative Potential of Polygonum cuspidatum Root Extracts," Journal of Natural Medicine, Vol. 64, No. 2, 2010, pp. 146-152. doi:10.1007/s11418-009-0387-8

[31] J. A. Shin, J. H. Shim, J. G. Jeon, K. H. Choi, E. S. Choi, N. P. Cho and S. D. Cho, "Apoptotic Effect of Polygonum cuspidatum in Oral Cancer Cells through the Regulation of Specificity Protein 1," Oral Diseases, Vol. 17, No. 2, 2011, pp. 162-170. doi:10.1111/j.1601-0825.2010.01710.x

[32] I. S. Lee, A. Nishikawa, F. Furukawa, K. Kasahara and S. U. Kim, "Effects of Selaginella tamariscina on in Vitro Tumor Cell Growth, P53 Expression, $\mathrm{G}_{1}$ Arrest and in Vivo Gastric Cell Proliferation," Cancer Letters, Vol. 144, No. 1, 1999, pp. 93-99. doi:10.1016/S0304-3835(99)00202-5

[33] E. Lee, K. K. Park, J. M. Lee, K. S. Chun, J. Y. Kang, S. S. Lee and Y. J. Surh, "Suppression of Mouse Skin Tumor Promotion and Induction of Apoptosis in HL-60 Cells by Alpinia oxyphylla Miquel (Zingiberaceae)," Carcinogenesis, Vol. 19, No.8, 1998, pp. 1377-1381. doi:10.1093/carcin/19.8.1377

[34] W. Ren and D. G. Tang, "Extract of Solanum muricatum (Pepino/CSG) Inhibits Tumor Growth by inducing Apoptosis," Anticancer Research, Vol. 19, No. 1, 1999, pp. 403-408.

[35] A. K. Taraphdar, M. Roy and R. K. Bhattacharya, "Natural Products as Inducers of Apoptosis: Implication for Cancer Therapy and Prevention," Current Science, Vol. 80, No. 11, 2001, pp. 1387-1396.

[36] S. M. Raja, S. Chen, P. Yue, T. M. Acker, B. Lefkove, J. L. Arbiser, F. R. Khuri and S. Y. Sun, "The Natural Product Honokiol Preferentially Inhibits Cellular FLICE-Inhibitory Protein and Augments Death Receptor-Induced Apoptosis," Molecular Cancer Therapeutics, Vol. 7, No. 7, 2008, pp. 2212-2223. doi:10.1158/1535-7163.MCT-07-2409

[37] H. Hasegawa, Y. Yamada, K. Komiyama, M. Hayashi, M. Ishibashi, T. Yoshida, T. Sakai, T. Koyano, T. S. Kam, K. Murata, K. Sugahara, K. Tsuruda, N. Akamatsu, K. Tsukasaki, M. Masuda, N. Takasu and S. Kamihira, "Dihydroflavonol BB-1, an Extract of Natural Plant Blumea balsamifera, Abrogates TRAIL Resistance in Leukemia Cells," Blood, Vol. 107, No. 2, 2006, pp. 679-688. doi:10.1182/blood-2005-05-1982

[38] M. Endo, S. Oyadomari, M. Suga, M. Mori and T. Gotoh, "The ER Stress Pathway Involving CHOP Is Activated in the Lungs of LPS-Treated Mice," Journal of Biochemistry, Vol. 138, No. 4, 2005, pp. 501-507. doi: $10.1093 / \mathrm{jb} / \mathrm{mvi143}$

[39] J. A. Shin, J. H. Shim, E. S. Choi, D. H. Leem, K. H. Kwon, S. O. Lee, S. Safe, N. P. Cho and S. D. Cho, "Chemopreventive Effects of Synthetic C-Substituted Diindolylmethanes Originating from Cruciferous Vegetables in Human Oral Cancer Cells," European Journal of Cancer Prevention, Vol. 20, No. 5, 2011, pp. 417-425. doi:10.1097/CEJ.0b013e32834473c3 